\title{
LE POISSON SENTINELLE DES MILIEUX AQUATIQUES : PERTINENCE ET OPTIMISATION DES INDICATEURS SANITAIRES.
}

\author{
P. GIRARD \\ Consultant Vétérinaire en Aquaculture et Environnement Aquatique, \\ $N^{\circ} 1$, lotissement Super-Peynier - 13790 Peynier (France).
}

\section{RÉSUMÉ}

Le Poisson est de plus en plus utilisé comme sentinelle de l'environnement et, aujourd'hui, de nombreuses méthodes, basées sur l'étude des communautés, sur les réponses physiologiques des individus et sur les réactions métaboliques et cellulaires font appel à lui. Parmi ces méthodes, l'évaluation de l'état sanitaire pourrait apparaître comme un indicateur pertinent de la qualité des milieux aquatiques.

Cependant, en raison de considérations d'ordre conceptuel et financier, peu d'indicateurs ont fait la preuve de leur pertinence. Ces indicateurs restent donc peu usités et ne sont qu'exceptionnellement intégrés dans les programmes de surveillance des milieux aquatiques.

Dans cet exposé, seront successivement développés, d'une part, les principaux paramètres sanitaires des poissons et leur signification écologique et, d'autre part, les limites actuelles de leur emploi et les moyens d'optimiser cet outil.

L'optimisation de l'outil sanitaire passe par la mise en place d'un réseau d'épidémiosurveillance, moyen de lutte contre les maladies basé sur l'observation et la prévention, dont le rôle consiste à structurer, organiser et coordonner les compétences spécifiques de chacun des acteurs de la filière et à assurer la circulation de l'ensemble des informations disponibles.

\section{FISH AS SENTINELS OF AQUATIC ENVIRONMENT : PERTINENCE AND OPTIMISATION OF SANITORY PARAMETERS.}

\section{ABSTRACT}

Fish are more and more used as proper sentinels of environment and many methods are now based upon the study of communities, physiological responses and metabolic and cellular reactions of fish. Among these methods, the evaluation of the health condition appears certainly as one of the most pertinent.

Nevertheless, by reason of understanding and financial motives, very few indicators have been proved to be really pertinent. These indicators are not widely practised and they are exceptionally integrated within biomonitoring programmes : in the reality of to-day, only the parasite Trichodina sp. has been validated and could be integrated in the "fish-index".

The present paper will first describe the main sanitory parameters and their ecological means and, secondly, so far their use is possible and the ways to improve the sanitory tool. 
The improvement of this tool implies to build up an epidemiological survey network in order to organize and coordinate the specific competencies and to connect all the available informations.

\section{INTRODUCTION}

Le poisson est de plus en plus utilisé comme sentinelle de l'environnement car il se révèle un excellent indicateur de la qualité des milieux aquatiques et, aujourd'hui, de nombreuses méthodes font appel à lui :

- méthodes basées sur l'étude des communautés : richesse, abondance, composition des espèces, structures démographiques intra- ou interspécifiques,

- méthodes basées sur les réponses physiologiques des individus : réactions comportementales, indices biométriques, reproduction, physiopathologie,

- méthodes utilisant les réactions métaboliques et cellulaires : réactions enzymatiques (activité EROD), quantification du stress, paramètres biochimiques sanguins : dosages hormonaux, hématocrite, formule-numération... (ALLARDI, 1994).

Parmi ces différents indicateurs, l'état sanitaire apparaît comme un indicateur pertinent de l'environnement, mais il reste peu usité et n'est qu'exceptionnellement intégré dans les programmes de surveillance des milieux aquatiques. Or, s'intéresser à la santé des écosystèmes sans prendre en compte la santé des poissons est difficilement concevable.

Aussi, dans cet exposé, seront successivement développés :

A. les principaux indicateurs sanitaires et leur signification écologique,

B. les limites actuelles de leur emploi et les moyens d'optimiser cet outil.

\section{A. LES PRINCIPAUX INDICATEURS SANITAIRES ET LEUR SIGNIFICATION ÉCOLOGIQUE}

Ils répondent à deux préoccupations complémentaires :

1. l'une prend en compte l'ensemble des modifications anatomopathologiques macroscopiques, mais dont la description ne renseigne pas sur les étiologies des processus observés, celles-ci étant soit in(mé)connues, soit, a contrario, multifactorielles : ce sont les indicateurs pathobiologiques,

2. l'autre vise à rechercher et à déterminer des agents potentiellement pathogènes dont la maladie est le plus souvent - mais pas toujours - l'expression, et dont la présence, liée à des conditions particulières du milieu, renseignera par conséquent sur l'état de ce milieu : ce sont les indicateurs pathobiotiques.

\section{LES INDICATEURS PATHOBIOLOGIQUES}

Les anomalies macroscopiques externes des poissons sont généralement l'expression d'altérations physiologiques ou biochimiques provoquées par la présence dans l'environnement de substances toxiques ou de bioagresseurs. Ces altérations morphologiques permettent d'évaluer les effets d'exposition de longues durées à de faibles 
niveaux de contamination, en particulier les lésions externes et celles de la colonne vertébrale ou d'organes comme le foie (LAFAURIE, 1995). Si les observations rapportées sont innombrables, elles se regroupent néanmoins en trois classes principales :

\subsection{Les déformations}

* du squelette : elles sont occasionnées essentiellement par la présence dans le milieu de pesticides et de métaux lourds (plomb, cadmium) (AFEE, 1976 ; ALDRIN, 1987 ; BOGE et al., 1991 ; DAUBA, 1980 ; LABAT et al., 1977 ; RAMADE, 1989). Des virus, des bactéries, des phénomènes héréditaires sont autant d'autres causes possibles (BRUSLE, 1995 ; MÖLLER, cité par ALDRIN, 1987).

* d'oeufs, d'embryons et de larves : le pourcentage de jeunes malformés, suite à l'exposition des gonades ou des oeufs à des substances toxiques, est souvent significatif de la contamination du milieu.

Les PCB, sur des oeufs et des embryons de lotes et de truites du lac Léman (BURGERMEISTER et al., 1983), le sulfate de fer chez des alevins de hareng Clupea harengus, le pétrole chez des alevins de plie, les retombées radioactives (DAVIS, 1970), les variations extrêmes des conditions de l'environnement, les déficits thermiques, la pollution chimique (MELLINGER, 1995), font partie des principales causes responsables des malformations rencontrées.

\subsection{Les lésions tissulaires}

Elles sont représentées par des érosions (des branchies, des nageoires), des ulcères (du tégument), des cataractes oculaires, des hyperplasies. Ces lésions sont caractéristiques de processus dégénératifs, prolifératifs ou infectieux, provoqués par le séjour prolongé des poissons dans des milieux pollués ou par des bioagresseurs : parasites trématodes dans le cas de cataractes, bactéries - type Flavobacterium $s p$. dans le cas d'atteintes cutanées (AFEE, 1978 ; ALDRIN, 1987 ; BERNARDET, 1989 ; BOGE et al., 1991 ; GHITTINO, 1966 ; RAMADE, 1989).

Le cadmium - nécroses dermiques chez l'anguille (GONY et al., 1988), érosions des nageoires de Limanda limanda et de Pleuronectes platessa (COSSA, 1989) -, le chrome ulcérations tégumentaires - et le pétrole et ses dérivés - ulcérations de $80 \%$ des échantillons de soles, de plies, de bars et de mulets pêchés après le naufrage de l'Amoco Cadiz (NOUNOU et al., 1980) -, représentent les trois principaux agents responsables de ces altérations cutanées.

\subsection{Les tumeurs}

De nombreuses études ont démontré que le nombre de poissons présentant des tumeurs augmente près des sources de pollution. Une étude sur la Fox River au Canada et aux USA révèle qu'en eaux polluées, $4,38 \%$ des poissons examinés (au nombre de 2 121) présentent des tumeurs, alors qu'en eau pure $1,03 \%$ des poissons examinés (au nombre de 4 369) en présentent (NIGRON, 1983).

Les substances le plus souvent incriminées sont : les Hydrocarbures Aromatiques Polycycliques (HAP), les Hydrocarbures Aliphatiques, les PCB, les Pesticides organiques, les Amines Aromatiques, les Ethers halogénés, et de nombreux métaux lourds : Arsenic, Beryllium, Cadmium, Chrome, Cobalt, Fer, Plomb, Nickel (ALDRIN, 1987 ; BOGE et al., 1991 ; RAMADE, 1989). 
Linfluence des saisons et des facteurs physico-chimiques du milieu : température oxygène dissous - salinité d'une part, de l'âge, du sexe, de la distribution géographique et du comportement trophique des poissons d'autre part, conditionne les niveaux de concentration et la pathogénie de la plupart des xénobiotiques:

- influence des saisons : pic saisonnier fin août-début septembre pour le lymphosarcome du brochet, prépondérance de la papillomatose de l'anguille du printemps à l'automne,

- influence de l'âge : hépatomes chez les truites arc-en-ciel de 3 à 4 ans, sensibilité maximale au lymphosarcome du brochet à l'âge de 4 ans, anguilles atlantiques de 3-4 ans plus touchées par la papillomatose,

- influence du sexe : chez les Poecilidae, les mâles présentent des tumeurs mélaniques plus fréquemment que les femelles,

- influence des facteurs physico-chimiques du milieu : température, oxygène dissous et, surtout, baisse de la salinité interviennent dans l'expression de la papillomatose de l'anguille,

- importance de la distribution géographique : les poissons sont dépendants de leur environnement et, par conséquent, certains néoplasmes apparaissent localisés géographiquement : papillomes des Pleuronectidae de la côte ouest de l'Amérique, lymphosarcome des Esocidae d'Irlande, papillomatose buccale des Anguillidae de la Baltique et de la Mer du Nord,

- des corrélations positives ont été démontrées entre l'apparition des papillomes buccaux et cutanés et le comportement de type fouisseur : seules les espèces benthiques - à l'exception du saumon atlantique - sont atteintes (NIGRON, 1983).

Toutefois, des virus (dans les papillomes cutanés des poissons plats et la papillomatose de l'anguille), des bactéries, des parasites, l'aflatoxine B1 (dans le cas des hépatomes de la truite arc-en-ciel), voire des hormones, sont impliqués ou participent, directement ou non, à ces processus tumoraux. Certains auteurs considèrent dans ce cas qu'il existe une relation indirecte avec la pollution : celle-ci serait un facteur de stress pour les poissons qui seraient alors plus sensibles aux agents pathogènes.

\subsection{Altérations diverses}

Des hémorragies cutanées chez des poissons tropicaux, sous l'action du cyanure servant à leur capture (HIGNETTE, 1984) et des lésions cornéennes chez des truites arc-en-ciel, provoquées par de l'étain (ALZIEU, 1989), ont également été signalées.

Pour certains auteurs, enfin, les indices biométriques - indice de condition et indices somatiques - offrent un certain intérêt pour évaluer les effets des pollutions chroniques car leurs modifications sont souvent l'expression d'altérations biochimiques ou physiologiques et s'avèrent être corrélées positivement avec le degré de pollution (BERRYMAN, 1990). Ces données doivent cependant être intégrées dans le contexte saisonnier et piscicole normal du cours d'eau étudié : cela implique l'existence de références sur l'état moyen de la population avant modification (WOLTERS, 1978).

\section{LES PARAMÈTRES BIOTIQUES}

Les pollutions organiques et minérales sont souvent impliquées, directement ou indirectement, dans l'apparition des processus pathologiques.

Le Tableau I ci-dessous recense quelques xénobiotiques connus pour induire ou favoriser le développement de certaines pathologies pisciaires. 


\section{Tableau I}

Recensement de quelques micropolluants connus pour induire certaines pathologies pisciaires.

\section{Table I \\ Census of some micropollutants known to induce different pathologies of wildfish.}

\begin{tabular}{|c|c|c|}
\hline AGENTS PATHOGENES & PATHOLOGIES & FACTEURS FAVORISANTS \\
\hline \multirow[t]{4}{*}{ PARASITES } & Saprolegniose & Phénols: NH3: Pesticides \\
\hline & Myxosporidiose & Insecticides (Sevin) \\
\hline & Trématodose & Heptachlore \\
\hline & ichtyophtiriose & Phenols : Polypropylene : $\mathrm{NH} 3$ \\
\hline \multirow[t]{5}{*}{ BACTERIES } & Furonculose & DDT $-\mathrm{NH} 3$ \\
\hline & Aèromonose à $H$. hydrophila & NH3 - Méthylmercure \\
\hline & Yersiniose & $\mathrm{Cu}^{++}-\mathrm{NH} 3$ \\
\hline & Vibriose à $V$. anguillarum & Cuivre- Hydrocarbures - $\mathrm{NH} 3$ \\
\hline & Pseudomonose & Méthylmercure \\
\hline \multirow[t]{4}{*}{ VIRUS } & Nécrose Hématopoiètique Infectieuse & Cuivre \\
\hline & Maladie lymphokystique & Hydrocarbures \\
\hline & Herpessvirose du turbot & Hydrocarbures \\
\hline & Papillomes et tumeurs & Hydrocarbures \\
\hline
\end{tabular}

Références (References) : (ANDREWS, GILES et BROWN, HETRICK et al., KNITTEL, LOWE, VLADIMIROV et FLEROV : in ALDRIN, 1987 ; DE KINKELIN et al., 1986 ; GIRARD, 1993 ; NICHOLSON, 1982).

Toutefois, la présence d'agents potentiellement pathogènes chez les poissons n'apporte que rarement la preuve du rôle des pollutions dans le déclenchement des processus pathologiques. En effet, seuls, à ce jour, quelques cas de corrélations entre pollutions et pathologies - et/ou présence d'agents pathogènes - ont pu être confirmés. Sont ainsi plus spécifiquement concernés :

\subsection{Les Parasites}

Parmi les agents potentiellement pathogènes révélateurs d'une pollution principalement organique - des milieux aquatiques, plusieurs genres parasitaires méritent une attention particulière en raison, d'une part, de leur représentativité et, d'autre part, de la facilité à les rechercher.

\subsubsection{Trichodina sp.}

Protozoaire cilié, Trichodina sp. est un parasite cutanéo-branchial peu pathogène des poissons. Une étude de surveillance sanitaire des populations piscicoles sauvages entreprise en 1995 sur le Réseau Hydrobiologique et Piscicole (R.H.P.) des régions Provence-Alpes-Côte d'Azur et Languedoc-Roussillon, a révélé sa présence préférentielle dans le mucus de poissons pêchés sur des stations perturbées soit par des rejets organiques, soit par des effluents de stations d'épuration. C'est pourquoi, une étude de validation de cet indicateur potentiel a été entreprise en 1996 avec l'objectif, à terme, de pouvoir intégrer Trichodina comme paramètre sanitaire dans l'indice-poisson. Cette étude a été réalisée sur des stations R.H.P. des régions Provence-Alpes-Côte d'Azur, Languedoc-Roussillon et Seine-Normandie (GIRARD, non publié, 1996).

Cette étude a consisté à comparer, par analyses graphiques, la qualité générale des eaux de rivières définie par l'approche Trichodina à différents paramètres physicochimiques du milieu, considérés soit individuellement (O2 dissous, \% 02, DBO5, MES, 
température, $\mathrm{NH} 4+$, classes de qualité), soit groupés (i.e. analyse multifactorielle). Les valeurs des différents paramètres physico-chimiques et des classes de qualité ont été communiquées par les DIREN ou les Agences de l'Eau concernées.

Sur chaque station prospectée, un nombre statistiquement significatif de poissons a été prélevé (dans tous les cas - sauf impossibilité majeure - : $n>30$ ). Les poissons analysés appartenaient aux espèces les plus représentatives des stations prospectées, et, pour la même espèce, deux classes d'âge ont été systématiquement examinées. Au total, 1935 poissons ont fait l'objet d'un examen parasitologique externe en vue de rechercher le parasite du genre Trichodina sp.

Les parasites ont été recherchés dans le mucus cutané des poissons. Celui-ci, après avoir été prélevé par grattage à l'aide d'une lame de bistouri et déposé entre lame et lamelle, a été observé au microscope optique (X 100).

Cinq classes de qualité parasitaire sont alors déterminées sur la base des critères présence/absence et abondance (ou charge) parasitaire. A chaque classe ainsi déterminée sont associées une notation, allant de 0 à +++, et une représentation chromatique. Une correspondance est ensuite établie avec les classes de qualité des eaux telles qu'elles sont définies par les Agences de l'Eau, conformément au Tableau II ci-dessous :

\section{Tableau II}

Abondance parasitaire et classes de qualité correspondantes telles qu'elles sont définies par les Agences de l'Eau.

\section{Table II}

Number of parasites and corresponding classes of quality as specified by French Water Agencies.

\begin{tabular}{|c|c|c|c|c|}
\hline Notation & Charge parasitaire & Equivalent & Classes Agence & Qualite de l'eau \\
\hline 0 & absence de parasites & 0 parasile & $1 \mathrm{~A}$ & excellente \\
\hline 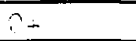 & atondance faible & a 10 narasites & $1 \mathrm{~B}$ & bonne \\
\hline$\ldots+$ & abondance forte & 51 a 100 parasites & 3 & médios:e \\
\hline$\overline{t++}$ & abondance très forte & $>100$ parasites & $\mathrm{HC}$ & mauvaise \\
\hline
\end{tabular}

Pour ce qui est des paramètres physico-chimiques, les résultats font apparaître sur les stations classées en qualité $1 \mathrm{~A}$ par les Agences une corrélation positive de l'ordre de 30 à $40 \%$, alors que sur toutes les stations classées en qualité $1 \mathrm{~B}, 2$ et 3 , les corrélations sont positives à plus de $80 \%$ entre la présence/abondance de Trichodina sp. et les différents paramètres considérés, soit respectivement :

- $83 \%$ pour l'oxygène dissous,

- $82 \%$ pour le taux de saturation en oxygène,

$-83 \%$ pour la DBO5,

$-83 \%$ pour les MES,

$-81 \%$ pour l'ammoniaque $\mathrm{NH}^{+}$,

- $90 \%$ pour la température. 
Pour ce qui est des classes de qualité d'eau définies par les Agences, les résultats de 1996 confirment ceux de 1995 et démontrent une corrélation positive voisine de $80 \%$ entre la présence et l'abondance de Trichodina sp., ainsi que le montre le Tableau III ci-dessous :

- la colonne 1 "Code " indique le code R.H.P. de la station concernée,

- la colonne 2 "Rivière " précise le cours d'eau prospecté,

- la colonne 3 "Cat " correspond à la catégorie du cours d'eau considéré,

- la colonne 4 "TRI " définit l'abondance parasitaire moyenne en Trichodines,

- la colonne 5 “Classe de qualité " détermine les classes de qualité d'après les critères Agence (AG) et Trichodina (TRI),

- la colonne 6 "Colorimétrie " propose une illustration chromatique des classes de qualité d'après les critères Agence (AG) et Trichodina (TRI).

\section{Tableau III}

Comparaison entre classes de qualité définies selon l'indice Trichodina sp. et les Agences de l'Eau sur 32 stations du réseau R.H.P.

\section{Table III}

Comparison between classes of quality according to Trichodina sp. index and Water Agencies on 32 stations belonging to R.H.P. network.

\begin{tabular}{|l|l|l|l|l|l|l|l|}
\hline Code & Rivière & Cat & TRI & \multicolumn{2}{|l|}{ Classe de qualité } & \multicolumn{2}{|c|}{ Colorimétrie } \\
\hline & & & & AG & TRI & AG & TRI \\
\hline 2702 & SEINE & $2 / G M$ & + & 3 & 2 & & \\
\hline 2703 & RISLE & 1 & $0+$ & $1 \mathrm{~B}$ & $1 \mathrm{~B}$ & & \\
\hline 2704 & EURE & 2 & $0+$ & $1 \mathrm{~B}$ & $1 \mathrm{~B}$ & & \\
\hline 2705 & ITON & 1 & $0+$ & $1 \mathrm{~B}$ & $1 \mathrm{~B}$ & & \\
\hline 2708 & EPTE & 1 & $0+$ & $1 \mathrm{~B}$ & $1 \mathrm{~B}$ & & \\
\hline 7501 & SEINE & $2 / \mathrm{GM}$ & + & 2 & 2 & & \\
\hline 7502 & SEINE & $2 / \mathrm{GM}$ & $0+$ & 2 & $1 \mathrm{~B}$ & & \\
\hline 7504 & REVEILLON & 2 & ++ & 3 & 3 & & \\
\hline 7602 & LEZARDE & 1 & $0+$ & $1 \mathrm{~B}$ & $1 \mathrm{~B}$ & & \\
\hline 7603 & CAILLIE & 1 & $0+$ & $1 \mathrm{~B}$ & $1 \mathrm{~B}$ & & \\
\hline 7605 & DURDENT & 1 & $0+$ & $1 \mathrm{~B}$ & $1 \mathrm{~B}$ & & \\
\hline 7606 & SCIE & 1 & $0+$ & $1 \mathrm{~B}$ & $1 \mathrm{~B}$ & & \\
\hline 7702 & YERRES & 2 & + & 2 & 2 & & \\
\hline 7705 & MARNE & $2 / \mathrm{GM}$ & $0+$ & 2 & $1 \mathrm{~B}$ & & \\
\hline 7706 & GD MORIN & 2 & $0+$ & $1 \mathrm{~B}$ & $1 \mathrm{~B}$ & & \\
\hline 7707 & OURCQ & 2 & 0 & $1 \mathrm{~B}$ & $1 \mathrm{~A}$ & & \\
\hline 0407 & DURANCE & 2 & $0+$ & $1 \mathrm{~B}$ & $1 \mathrm{~B}$ & & \\
\hline 0408 & DURANCE & 2 & $0+$ & $1 \mathrm{~B}$ & $1 \mathrm{~B}$ & & \\
\hline 0505 & DURANCE & 1 & 0 & $1 \mathrm{~B}$ & $1 \mathrm{~A}$ & & \\
\hline 0601 & BEVERA & 1 & 0 & $1 \mathrm{~A}$ & $1 \mathrm{~A}$ & & \\
\hline 0602 & LOUP & 1 & $0+$ & $1 \mathrm{~B}$ & $1 \mathrm{~B}$ & & \\
\hline 0605 & VAR & 1 & $0+$ & $1 \mathrm{~B}$ & $1 \mathrm{~B}$ & & \\
\hline 1301 & TOULOUBRE & 2 & $0+$ & 3 & $1 \mathrm{~B}$ & & \\
\hline 1302 & REAL & 1 & $0+$ & $1 \mathrm{~B}$ & $1 \mathrm{~B}$ & & \\
\hline 1303 & DURANCE & 2 & $0+$ & $1 \mathrm{~B}$ & $1 \mathrm{~B}$ & & \\
\hline 1304 & ARCI & 2 & $0+$ & $1 \mathrm{~B}$ & $1 \mathrm{~B}$ & & \\
\hline 1305 & ARC II & 2 & $0+$ & 3 & $1 \mathrm{~B}$ & & \\
\hline 3403 & HERAULT & $2 / \mathrm{GM}$ & $0+$ & $1 \mathrm{~B}$ & $1 \mathrm{~B}$ & & \\
\hline 3404 & VIS & 1 & 0 & $1 \mathrm{~A}$ & $1 \mathrm{~A}$ & & \\
\hline 4804 & LOT & 1 & 0 & $1 \mathrm{~A}$ & $1 \mathrm{~A}$ & & \\
\hline 4806 & CHAPEAUROUX & 1 & $0+$ & $1 \mathrm{~B}$ & $1 \mathrm{~B}$ & & \\
\hline 4807 & TRUYERE & 1 & 0 & $1 \mathrm{~A}$ & $1 \mathrm{~A}$ & & \\
\hline
\end{tabular}


Par ailleurs, l'indice Trichodina permet de suivre l'évolution temporelle de la qualité des milieux. Ainsi, l'étude de validation réalisée a permis de comparer 22 stations suivies en 1995 et 1996 : il en est ressorti une amélioration sur 6 stations, une dégradation sur 4 stations et un statu quo sur 12 stations.

L'exemple ci-dessous, s'adressant à cinq stations R.H.P. situées dans le département des Bouches-du-Rhône, illustre cette approche. II permet en effet d'apprécier entre 1995 et 1996 :

- une amélioration sur les stations : 1301 (Touloubre) passage de la classe 2 à 1B, 1305 (Arc aval) passage de la classe 2 à $1 B$,

- une dégradation sur la station : 1304 (Arc amont) passage de la classe $1 \mathrm{~A}$ à 1B,

- une stabilité sur les stations: 1302 (Réal) classe de qualité $1 \mathrm{~B}$, 1303 (Durance) classe de qualité 1B.

La représentation graphique de cet exemple est matérialisée par le diagramme cidessous (Figure 1):

Année 1995

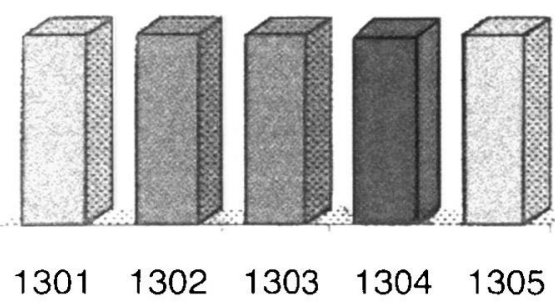

Année 1996

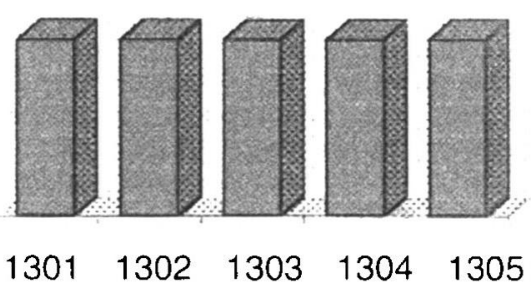

\section{Figure 1}

Etude comparative des classes de qualité selon l'indice Trichodina sur cinq stations R.H.P. des Bouches-du-Rhône.

\section{Figure 1}

Comparative study of classes of quality according to Trichodina index on five stations belonging to R.H.P. network in the department "Bouches-du-Rhône ".

La présence des Trichodines paraît donc hautement corrélée à la dégradation plus ou moins importante du milieu, tant en termes de paramètres physico-chimiques pris individuellement qu'en termes de qualité des eaux. Sur la base des critères présence et abondance, le parasite Trichodina peut donc être considéré comme un indicateur pertinent de la qualité générale des milieux aquatiques (GIRARD, non publié, 1996).

\subsubsection{Epistylis, Ambiphrya, Glossatella}

Ces trois protozoaires ciliés appartiennent au sous-ordre des Sessilina et présentent des analogies morphologiques et structurales très proches. Peu pathogènes, ils s'observent dans le mucus cutané et branchial des poissons qui vivent dans des milieux riches en matière organique. Ils sont généralement considérés comme des indicateurs potentiels de contamination fécale (CHAMPIAT et LARPENT, 1988). 


\subsubsection{Eimeria sp.}

Appartenant à la sous-classe des Coccidies, ce parasite interne, généralement intestinal, est fortement pathogène. II apparaît également comme un indicateur potentiel de contamination fécale (CHAMPIAT et LARPENT, 1988).

Par ailleurs, une synergie entre pollution et parasitose a été observée dans les sites les plus pollués de la Weser dans lesquels les taux de prévalence des infestations d'anguilles par des Acanthocéphales et des Nématodes sont les plus élevés (BRUSLE, 1994).

\subsection{Les Bactéries}

De nombreuses bactéries, plus ou moins ichtyopathogènes, profitent de certaines conditions environnementales particulières pour se développer. Mais la plus représentative d'entre elles est certainement Clostridium botulinum, responsable du botulisme.

Clostridium botulinum type $\mathrm{E}$, responsable du botulisme pisciaire, est un bacille tellurique, gram + , anaérobie strict, présent dans le sol, l'eau et les sédiments. Les spores sont concentrées dans les grandes réserves d'eau des continents (grands lacs) et dans les sédiments marins voisins des côtes par suite de l'érosion par ravinement. On peut aussi isoler les spores du contenu intestinal des vertébrés - poissons, oiseaux et mammifères et des invertébrés chez lesquels elles peuvent transiter. Par ailleurs, des analyses effectuées en novembre et décembre 1995 sur des invertébrés et des poissons du lac de Créteil ont révélé la présence de toxine de type $E$ dans la chair de bivalves, d'écrevisses et de poissons-chats (BEELE, 1996).

La toxine se caractérise par une persistance durable dans les organismes végétaux ou animaux vivant dans les plans d'eau contaminés lors des épizooties estivales, les plans d'eau à hauts risques étant ceux qui présentent une forte charge en matières organiques et un appauvrissement en $\mathrm{O} 2$. Par contre, les risques sont plus faibles dans des eaux à $\mathrm{pH}$ acide (VEYRET, 1986).

Maladie d'actualité se traduisant généralement par une toxiinfection alimentaire grave atteignant les animaux et l'homme (principalement les types A, B et E) - (POPOFF et ARGENTE, 1996) - le botulisme pisciaire est avant tout une maladie de l'environnement et, principalement, des zones humides. Même si on la rencontre aussi parfois au sein d'élevages, par exemple au Danemark, où jusqu'à $82 \%$ des poissons d'élevage sont contaminés, en Italie ou en Grande-Bretagne (CANN et TAYLOR, 1982), elle affecte essentiellement l'avifaune, les oiseaux aquatiques (anatidés, oiseaux piscivores) étant des cibles particulièrement vulnérables.

\subsection{Les Virus}

La maladie lymphokystique de la limande et du flet, dont la prévalence est significativement plus élevée chez les poissons provenant de zones polluées (LEBRUN, 1990), et la papillomatose de l'anguille semblent être les deux seules viroses réellement induites par la pollution des milieux aquatiques.

\section{B. LES LIMITES ACTUELLES ET LES PERSPECTIVES D'OPTIMISATION}

"La prévalence des maladies des poissons sauvages est fortement influencée par les facteurs naturels : alimentation, variations de salinité, de température, pression de sélection par les prédateurs, autant de facteurs qui sont souvent négligés par des enquêteurs obnubilés par la pollution " (MÖLLER, in ALDRIN, 1987). Cette tendance systématique à trop vouloir corréler la présence d'un agent pathogène, voire une 
manifestation pathologique, à une pollution ou à un xénobiotique précis, occulte en effet le rôle et l'incidence des phénomènes naturels, tels les fluctuations thermiques, les blooms algaux et les surdensités, qui sont souvent considérés comme des facteurs déclenchants (BUCKE, 1997).

\section{PERTINENCE ET LIMITES ACTUELLES}

\subsection{Pertinence des indicateurs sanitaires}

\subsubsection{Les indicateurs spécifiques}

Parmi tous les indicateurs sanitaires, Trichodina sp., et, à un degré moindre, les Sessilina et le botulisme pisciaire, sont quasiment les seuls à être représentatifs d'une situation environnementale donnée et susceptibles de renseigner sur la nature de la pollution : charge organique des milieux par Trichodina, contamination fécale par les Sessilina, et sédimentation - voire envasement - et anaérobiose des milieux par Clostridium botulinum type $E$.

\subsubsection{Les indicateurs non spécifiques}

Représentant la majorité des indicateurs - altérations macroscopiques externes, pathologies - ils ne renseignent en aucune manière sur la nature des perturbations responsables. Toutefois, on peut considérer, a priori, que leur prévalence d'une part, les écarts aux états de référence d'autre part, sont souvent liés, voire proportionnels, au degré de pollution et à l'atteinte des milieux.

Pour l'heure, en l'absence d'études de validation, la pertinence de ces indicateurs n'est pas démontrée et leur intérêt se limite donc à apprécier la tendance globale de la qualité des milieux aquatiques et réside principalement dans leur capacité à déclencher des signaux d'alerte et à souligner toute modification environnementale éventuelle : "Clinical diseases are recorded in pollution studies as biological indicators of environmental change » (BUCKE, 1997).

Aussi et en l'état actuel des connaissances, il paraît raisonnable de limiter l'utilisation des indicateurs sanitaires aux quelques paramètres bien appréhendés.

\subsection{Limites actuelles}

Probablement liées au manque d'intérêt suscité par les problèmes ichtyosanitaires et aux difficultés d'interprétation des processus pathologiques, elles sont de trois ordres:

- confidentialité et dispersion des informations existantes,

- manque de communication et de coordination au sein de la filière (acteurs de terrain, gestionnaires, biologistes),

- insuffisance - voire absence - d'études écopathologiques et d'expérimentations destinées à valider les approches sanitaires.

\section{MOYENS D'OPTIMISATION}

Nécessaires, ils s'appuient sur quatre axes : sélection, hiérarchisation, objectivation et validation des indicateurs sanitaires. 


\subsection{La sélection des indicateurs}

Ne méritent d'être retenus comme indicateurs que ceux dont la pertinence, la spécificité et la représentativité sont prouvées, certes, mais qui répondent également aux critères suivants :

- facilité de mise en oeuvre, avec contrôles en routine,

- corrélations avec d'autres indicateurs déjà utilisés,

- coût faible.

Seuls, parmi les indicateurs que nous avons passés en revue, le parasitisme cutané à ciliés (dont Trichodina $s p$. et les Sessilina), l'agent du botulisme pisciaire et, dans une moindre mesure, les indicateurs pathobiologigues, répondent positivement à l'ensemble de ces critères, ce qui n'est pas le cas actuellement des agents pathogènes responsables des pathologies parasitaires, bactériennes ou virales déclarées.

En conséquence, l'intégration du parasite Trichodina sp. dans l'indice-poisson comme paramètre sanitaire paraît tout à fait réalisable dans la mesure où les principaux critères auxquels un indice doit répondre - pertinence, reproductibilité, fiabilité, rapidité, simplicité - sont assurés.

\subsection{L'objectivation des données}

L'objectivation des données disponibles est une nécessité qui suppose :

a) de les rassembler et de les synthétiser,

b) de les affiner en entreprenant des enquêtes épidémiologiques.

Pour ce faire, seul un réseau d'épidémiosurveillance, outil de lutte contre les maladies pisciaires basé sur l'observation et la prévention, peut répondre à cette attente. Un tel réseau se définit par :

* ses objectifs : ils consistent à assurer une surveillance rigoureuse et permanente, dans le temps et dans l'espace, des pathologies existantes et à garantir la qualité sanitaire des espèces piscicoles en prévenant l'introduction et la dissémination dans les milieux aquatiques d'agents pathogènes exogènes et de micropolluants,

* ses missions : elles consistent à surveiller l'évolution des pathologies existantes et l'émergence de nouvelles maladies, à recenser les espèces piscicoles sensibles et les zones à risques ou à surveillance prioritaire, à veiller à l'application des mesures prophylactiques, réglementaires ou non, à procéder aux prélèvements de terrain destinés aux laboratoires d'analyses, à recenser, interpréter et diffuser toutes les informations utiles et, enfin, à initier, coordonner et budgétiser toutes les actions visant à réduire les risques pathologiques,

* son organisation : elle est à double étage et repose : en amont, sur un observatoire national des risques, composé d'un bureau permanent et d'un collège constitué de scientifiques, de gestionnaires, de professionnels et des administrations compétentes et, en aval, sur des réseaux d'observation loco-régionaux, constitué de professionnels de terrain : Gardes-pêche, Agents des Services de l'Environnement, Laboratoires Vétérinaires Départementaux.

\subsection{L'interprétation des données}

L'interprétation des données repose sur la hiérarchisation des différents indicateurs qui s'établit sur le modèle des grilles de qualité. Pour chacun des paramètres susceptibles d'être retenus, cinq classes de qualité sont déterminées, correspondant : 
- aux classes d'abondance pour la charge parasitaire comme cela a été précisé pour le parasite Trichodina sp.,

- aux taux de prévalence pour les altérations externes et les pathologies.

Les classes de qualité pourraient s'établir selon le modèle représenté dans le Tableau IV ci-dessous et inspiré des classes de qualité proposées par OBERDORFF pour la mise au point de l'Indice d'Intégrité Biotique (OBERDORFF et HUGHES, 1992) :

\section{Tableau IV}

Correspondance entre taux de prévalence et classes de qualité "Agences de l'Eau ".

\section{Table IV}

Corresponding between rates of prevalence and classes of quality "Water Agencies".

\begin{tabular}{|l|l|c|l|}
\hline \multicolumn{1}{l}{ Classes } & Prévalence & \multicolumn{1}{c|}{ Classes Agence } & Qualité de l'eau \\
\hline $0-1 \%$ & non significatif & $1 \mathrm{~A}$ & excellente \\
\hline $1-5 \%$ & faible & $1 \mathrm{~B}$ & bonne \\
\hline & & & \\
\hline $20-35 \%$ & forte & 3 & médiocre \\
\hline$>35 \%$ & très forte & $\mathrm{HC}$ & mauvaise \\
\hline
\end{tabular}

\subsection{La validation}

Pour l'heure, nous l'avons souligné précédemment, seul le parasite cilié Trichodina sp. a fait l'objet d'une étude de validation. Celle d'autres indicateurs sanitaires mérite aussi d'être entreprise et doit reposer sur :

- des études épidémiologiques de terrain et des expérimentations de laboratoire,

- le recours à des moyens de diagnostic complémentaires, telles la microscopie électronique et l'histopathologie,

- des corrélations avec d'autres paramètres:

- biotiques (IBGN, indices diatomiques, IBI), xénobiotiques).

- abiotiques (physico-chimie, teneurs des bryophytes et du sédiment en

Ces paramètres biotiques et abiotiques comprennent tous les facteurs susceptibles d'intervenir dans l'apparition et le développement des bioagresseurs responsables des processus pathologiques:

- les paramètres physico-chimiques: amplitudes thermiques, oxygène dissous, débits, $\mathrm{pH}$, dureté, etc.,

- la capacité trophique des milieux,

- les sources de pollutions (physiques, organiques, chimiques),

- les espèces piscicoles présentes (densités, classes d'âge), entretien,

- les modalités de gestion halieutique des milieux : introductions, repeuplements,

- les usages de l'eau, autres que halieutiques. 


\section{CONCLUSION}

On dispose d'une multitude de faits d'observation, de présomptions, voire d'informations, sur les corrélations existant entre la qualité des milieux aquatiques et l'état sanitaire des poissons, mais les preuves de ces corrélations n'ont jamais été réellement établies et leur validation exceptionnellement réalisée. En fait, cela tient essentiellement à des considérations d'ordre conceptuel (CAIRNS, in BERRYMAN, 1990) et financier (BUCKE, 1997). Par ailleurs, les poissons marins ont fait l'objet d'études sanitaires bien plus nombreuses et approfondies que ceux d'eau douce.

Par conséquent, en milieux dulçaquicoles, le recensement, la centralisation et l'objectivation de l'ensemble des informations disponibles et la réalisation de programmes de recherches, principalement en matière d'épidémiologie, doivent être entrepris dans le cadre d'un réseau d'épidémiosurveillance dont la création s'avère désormais indispensable.

En effet, au même titre que les enquêtes épidémiologiques réalisées dans le cadre de programmes d'études spécifiques - programme E.L.P.E.M.M. (Etude des Lésions des Poissons et Mammifères Marins), placé sous l'égide de l'IFREMER et effectué entre 1978 et 1984, groupe de travail "Pathologie des animaux marins " au Conseil International pour l'Exploration de la Mer (C.I.E.M.) -, l'importance et l'efficacité des réseaux d'épidémiosurveillance ne sont plus à démontrer, à l'image soit des réseaux de surveillance français déjà constitués et opérationnels : réseau SAGIR, piloté par l'Office National de la Chasse (O.N.C.), Réseau de surveillance microbiologique (REMI), réseaux de surveillance de certaines pathologies animales (brucellose, salmonelloses, rage, etc.), soit des réseaux étrangers (au Canada, notamment au Québec, et aux U.S.A.) dont la particularité consiste à intégrer systématiquement les poissons dans les programmes de surveillance des écosystèmes aquatiques.

A terme, un réseau d'épidémiosurveillance a un double objectif : d'abord celui de poser le diagnostic de l'état sanitaire des milieux aquatiques en raison de la représentativité de certains agents pathogènes vis-à-vis de la qualité de ces milieux (agents responsables de : certaines viroses, trichodinose, botulisme), et, ensuite et surtout, celui de pouvoir mieux appréhender l'étiologie et l'épidémiologie de certaines pathologies devant faire l'objet de surveillances particulières en raison de l'intérêt majeur qu'elles présentent sur les plans économique, écologique et sanitaire :

- risques économiques : rhabdoviroses (S.H.V., N.H.I.),

- menaces de régression, voire d'extinction, qu'elles font peser sur les populations piscicoles sauvages : bucéphalose larvaire, aphanomycose, anguillicolose, pollutions organiques et métalliques,

- risques liés à la consommation de poissons : contaminations, zoonoses.

Et comme il ne peut y avoir de gestion piscicole et halieutique sans gestion sanitaire, gardons à l'esprit, en plagiant JUVENAL, la pertinence de cet adage : "Piscis sanus in aqua sana".

\section{BIBLIOGRAPHIE}

AFEE (Association Française pour l'Etude des Eaux), 1976 et 1978. Extraits de synthèses bibliographiques :

- rapport $n^{\circ} 3$ : le plomb, l'étain, 1976, 58-67.

- rapport $n^{\circ} 4$ : le zinc, le cadmium, 1978,60-73. 
ALDRIN J.F., 1987. Pollution chimique et sensibilisation des poissons aux agents pathogènes. Ichtyophysiologica Acta, Tome 11, 164-186.

ALLARDI J., 1994. Les poissons comme "indicateur biologique " de l'état des écosystèmes aquatiques. In Les variables biologiques: des indicateurs de l'état de santé des écosystèmes aquatiques. Séminaire National, texte des conférences, Ministère de l'Environnement, $233 \mathrm{p}$.

ALZIEU C., 1989. L'étain et les organoétains. Biogéochimie et écotoxicologie. Ifremer, rapports scientifiques et techniques, $N^{\circ} 17,93 p$.

BEELE X., 1996. Maîtrise du botulisme hydrique : l'implication des vétérinaires sapeurspompiers. La Dépêche Vétérinaire, 476, 2 pages.

BERNARDET J.F., 1989. Les flexibactérioses des poissons : deux aspects d'infections à Cytophagales récemment identifiées en France. La Pisciculture Française, 96, 1924.

BERRYMAN D., 1990. Sélection de nouveaux indicateurs de la qualité des cours d'eau du Québec. Ministère de l'Environnement de Québec, Direction de la qualité des cours d'eau, QE-90-05, 21-24 et 40-42.

BOGE G., ROCHE H., HOUVET D., 1991. Les indicateurs physiologiques de toxicité en milieu marin. Océanis, Vol. 17, Fasc. 4, 351-365.

BRUSLE J., 1994. L'anguille européenne, Anguilla anguilla, un poisson sensible aux stress environnementaux et vulnérable à diverses atteintes pathogènes. Bull. Fr. Pêche Piscic., 335, 237-260.

BRUSLE J., 1995. Skeletal abnormalities in fish and their multifactorial aetiology : a review. In E.A.F.P., seventh international conference "Diseases of fish and shellfish ", p. 90.

BUCKE D., 1997. Facts and myths regarding pollution and fish health. Bull. Eur. Ass. Fish Pathol., 17(6), 191-196.

BURGERMEISTER G., BEDRANI M., TARRADELLAS J., 1983. Utilisation de la lote Lota lota comme indicateur de la pollution des eaux continentales par des polluants organochlorés. Eau du Québec, Vol. 16, $n^{\circ} 2,135-143$.

CANN D.C., TAYLOR L.Y., 1982. An outbreak of botulism in rainbow trout, Salmo gairdneri Richardson, farmed in Britain. Journal of Fish Diseases, 5, 393-399.

CHAMPIAT D., LARPENT J.P., 1988. Biologie des eaux : méthodes et techniques. Masson, Paris, $374 \mathrm{p}$.

COSSA D., LASSUS P., 1989. Le cadmium en milieu marin. Biogéochimie et écotoxicologie. Ifremer, rapports scientifiques et techniques, $N^{\circ} 16,111 p$.

DAUBA F., 1980. Influence des métaux sur le milieu aquatique, synthèse bibliographique. 9-24.

DAVIS C.C., 1970. The effects of pollutants on the reproduction of marine organism. In FAO Technical Conference on marine pollution and its effects on living resources and fishing, 9-18 December 1970, Rome, 1-13.

DE KINKELIN P., MICHEL C., GHITTINO P., 1986. Précis de Pathologie des Poissons. Office International des Epizooties, Institut National de la Recherche Agronomique, Paris, $348 p$.

GHITTINO P., 1966. Les maladies des poissons et les dommages causés aux poissons en Italie par des facteurs ambiants défavorables. Bull. Off. Int. Epiz., 65, (5-6), 583588.

GIRARD P., 1993. Conséquences des pollutions sur les poissons, synthèse bibliographique. Aquarevue, 49, 29-33. 
GIRARD P., 1996. Validation du parasitisme à Trichodina sp. en vue de son intégration comme indicateur sanitaire dans l'indice-poisson. Etude CSP/Inter-Agences, convention $n^{\circ}$ 96195. Rapport final non publié, $34 \mathrm{p} .+$ annexes.

GONY S., LECOMTE-FINIGER R., FAGUET D., BIAGIANTI S., BRUSLE J., 1988. Etude expérimentale de l'action du cadmium sur les juvéniles d'anguille : biologie du développement et cytopathologie. Océanis, Vol. 14, Fasc. 1, 141-148.

HIGNETTE M., 1984. Méthodes de diagnostic de l'intoxication des poissons au cyanure. In "Utilisation du cyanure pour la capture des poissons". Océanis, Vol. 10, Fasc. 5, 585-591.

LABAT R., ROQUEPLO C., RICARD J.M., LIM P., BURGAT M., 1977. Actions écotoxicologiques de certains métaux $(\mathrm{Cu}, \mathrm{Zn}, \mathrm{Pb}, \mathrm{Cd})$ chez les poissons dulçaquicoles de la rivière Lot. Ann. Limnol., 13 (2), 191-207.

LAFAURIE M., 1995. Etat des connaissances opérationnelles sur la contamination et les indicateurs de pollution chimique toxique du milieu marin : 3 . Biomarqueurs, synthèse. Comité Scientifique Littoral Méditerranéen, $67 \mathrm{p}$.

LEBRUN Ph., 1990. Lésions macroscopiques relevées à la criée (enquête à Cherbourg sur quatre espèces piscicoles). Th. Doc. Vet., Nantes, $171 \mathrm{p}$.

MELLINGER J., 1995. Malformations embryonnaires et larvaires dues à la pollution marine. Mise au point bibliographique. Ichtyophysiologica Acta, 18, 145-152.

NICHOLSON B.L., 1982. Infectious Hematopoïetic Necrosis (I.H.N.). Actes du Symposium International de Talloires : "Les antigènes des micro-organismes pathogènes des poissons ". Collection Fondation Marcel Mérieux, 63-78.

NIGRON P., 1983. Tumeurs et cancérogénèse chez les poissons. Th. Med. Vet., Faculté de Lyon, $186 \mathrm{p}$.

NOUNOU P., MARTOJA R., ORCEL L., 1980. Ulcérations des poissons et mammifères marins pêchés dans les eaux côtières françaises. Publications du CNEXO, rapports scientifiques et techniques, $\mathrm{N}^{\circ} 43,94 \mathrm{p}$.

OBERDORFF T., HUGHES R.M., 1992. Modification of an index of biotic integrity based on fish assemblages to characterize rivers of the Seine Basin, France. Hydrobiologia, 228, 117-130.

POPOFF M.R., ARGENTE G., 1996. Le botulisme animal est-il une menace pour l'homme ? Bull. Acad. Vet. de France, 69, 373-382.

RAMADE F., 1989. Eléments d'écologie : écologie appliquée. Mac Graw-Hill, 578 p.

VEYRET M.F., 1986. Le botulisme pisciaire. Th. Med. Vet., Faculté de Médecine de Créteil, $94 \mathrm{p}$.

WOLTERS G.N., 1978. Ecologie des petits salmonidés sédentaires ; modifications de leur écosystème et ses conséquences. Th. Doc. Vet., Université de Toulouse, $136 \mathrm{p}$. 